\title{
Why nature has elected Michaelis-Menten kinetics for enzymes: a tentative rationale from variational calculus
}

\author{
by ANA P. CARVALHO and F. XAVIER MALCATA \\ Escola Superior de Biotecnologia, Universidade Católica Portuguesa, Rua Dr. António \\ Bernardino de Almeida, 4200 Porto, Portugal
}

\begin{abstract}
The kinetic performance of enzymes, the catalysts designed by nature to accelerate the chemical reactions that support life, has traditionally been described in terms of a rate expression first derived by Michaelis and Menten in the beginning of this century. Why nature has selected such kinetic behaviour remains, however, a mystery. A tentative rationale based on Euler's equation was developed and, after having eliminated functional forms due to physicochemical unfeasibility, a final open-form objective function (written as an infinite series and including dependencies on the substrate concentration, on the reaction rate, and on the derivative thereof with respect to concentration) is found. The integral of such an objective function is maximized by MichaelisMenten kinetics and yields its maximum value when the upper integration limit is roughly equal to the Michaelis-Menten constant.
\end{abstract}

\section{Introduction}

Enzymes are globular proteins with catalytic properties, i.e. enzymes bring about increases in the rate of biochemical reactions without undergoing a permanent chemical change [1]. Such increases are obtained, under mild environmental conditions, at the expense of lowering the activation energies of said reactions via more favourable reactional mechanisms. Enzymes are of universal occurrence in biological materials and life itself depends on a complex network of chemical reactions brought about by specific enzymes [2]. Another unique feature of enzymes with respect to inorganic catalysts is their high specificity towards substrates, which is thought to be a consequence of their elaborate, energydependent three-dimensional architecture. Enzyme studies have enjoyed remarkable growth in recent years because of their ever growing importance in scientific and technological terms; enzymology has permitted relevant contributions to such widespread activities as brewing, food flavour additives, pest control, dry cleaning, detergents, and analytical determinations [2].

Since the pioneering work of Michaelis and Menten back in 1913, the catalytic performance of enzymes has been widely described by a rectangular hyperbolic function of substrate concentration that departs from first-order behaviour at nil concentration and eventually approaches a horizontal asymptote at saturating concentrations. Knowing that nature tends to evolve so as to optimize its own resources, as would be predicted by Darwinistic approaches, one would expect the Michaelis-Menten rate expression to provide the best compromise between various intrinsic factors on the cellular level that might play a role in biochemical reactions in vivo. 
It is well established that enzymes possess two major features as biocatalysts, namely their activity and their controllability. The former relates to the high rates observed for enzyme-catalysed reactions when compared with the uncatalysed reaction counterpart. The latter may be substantiated by the capacity the enzyme possesses to modulate its own activity in response to microenvironmental factors, namely via substrate and/or product inhibition and heterologous activation [3]. It is therefore reasonable to expect that educated attempts to find an objective function that nature might have tried to optimize should encompass the concentration of substrate, $C$ (i.e. the intrinsic cell processing variable), the reaction rate, $r$ (i.e. the measure of the enzyme activity), and the derivative of the latter with respect to the former, $r^{\prime}$ (i.e. the measure of the enzyme controllability) as variables. On the other hand, the whole physiological range of living activity of the cell should be considered, namely in terms of an average behaviour, and so the integral of the objective function extended to the range of concentrations of interest would to advantage be considered rather than a simple, point value located somewhere in that range. It is the purpose of this work to formally build an objective function along these lines that meets the constraints of biochemical performance and leads to the Michaelis-Menten rate expression as the functional optimal form.

\section{Mathematical analysis}

In order to facilitate the analysis, dimensionless (and normalized, wherever possible) variables will be used hereafter. Recalling the form of Michaelis-Menten rate expression, the dimensionless concentration, rate, and derivative of the rate with respect to concentration may be written as

$$
\begin{gathered}
C^{*}=\frac{C}{K_{\mathrm{m}}} \quad\left(0 \leqslant C^{*}\right) \\
r^{*}=\frac{r}{v_{\max }}=\frac{C^{*}}{1+C^{*}} \quad\left(0 \leqslant r^{*} \leqslant 1\right)
\end{gathered}
$$

and

$$
r^{\prime *}=\frac{r^{\prime}}{\frac{v_{\max }}{K_{\mathrm{m}}}}=\frac{1}{\left(1+C^{*}\right)^{2}} \quad\left(0 \leqslant r^{\prime *} \leqslant 1\right)
$$

respectively, where $C, r$, and $r^{\prime}$ denote the substrate concentration, the reaction rate, and the derivative of the latter with respect to the former, $K_{\mathrm{m}}$ is the Michaelis-Menten constant, and $v_{\max }$ is the maximum reaction rate. The variation of $r^{*}$ and $r^{*}$ are depicted in Figure 1.

Using equations (1)-(3), the integral of the objective function introduced previously will read

$$
I\left[r^{*}\right]=\frac{\int_{0}^{C_{\max }^{*}} f\left\{C^{*}, r^{*}, r^{* *}\right\} \mathrm{d} C^{*}}{C_{\max }^{*}}
$$

where $I$ is a functional of $r^{*}$, and $C_{\max }^{*}$ is the upper limit of the concentration range of interest; the goal of the present analysis is thus to choose the function $f$ so that 


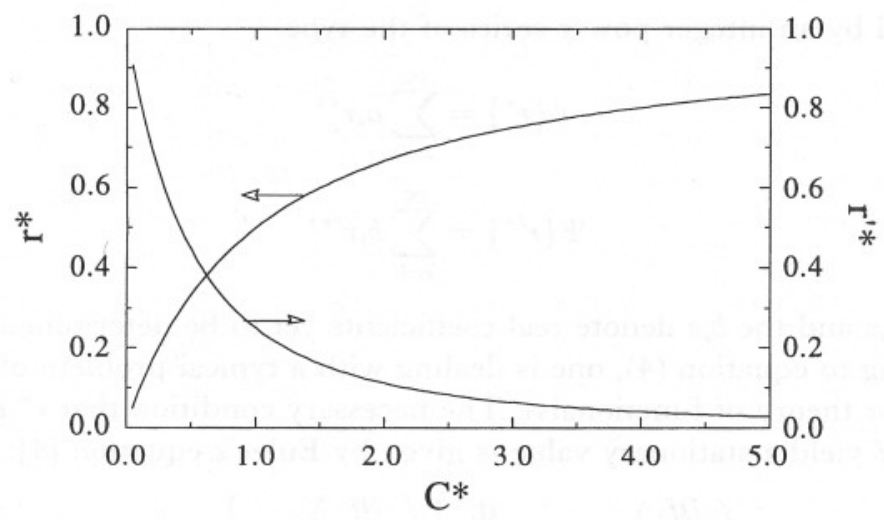

Figure 1. Variation of the dimensionless rate, $r^{*}$, and the dimensionless derivative of the rate with respect to the substrate concentration, $r^{\prime *}$, versus the dimensionless substrate concentration, $C^{*}$.

the functional has a maximum value. Clearly there are several forms of $r^{*}=r^{*}\left\{C^{*}\right\}$ that yield a maximum for the above functional; however, most of them violate physicochemical constraints and are therefore not eligible for our purpose.

In view of previous considerations, it is logic to postulate that the contributions from the rate, on the one side, and the derivative thereof, on the other, should be somewhat combined in the integrand function $f$; the simplest way of doing so is by adding such two contributions. On the other hand, the weight ascribed to each of those functions should depend on the actual concentration. Using these postulates, the integrand in equation (4) tentatively becomes

$$
f\left\{C^{*}, r^{*}, r^{*}\right\}=\phi\left\{C^{*}\right\} \psi\left\{r^{*}\right\}+\Phi\left\{C^{*}\right\} \Psi\left\{r^{\prime *}\right\}
$$

where $\phi$ and $\Phi$ are univariate functions of $C^{*}, \psi$ is a univariate function of $r^{*}$, and $\Psi$ is a univariate function of $r^{\prime *}$.

Each univariate function playing the role of a weight parameter may, for the sake of simplicity, be considered to be well described by a power function. Furthermore, it is convenient that (i) from the point of view of efficiency of enzymatic action, the substrate molecules should be consumed as fast as possible, with this issue being of particular relevance for low concentrations of substrate (i.e. $\phi$ should take high values for low $C^{*}$ and low values for high $C^{*}$ ), and that (ii) from the point of view of efficiency of the intrinsic concentration-based control mechanism of enzymatic action, a small variation in the concentration of substrate molecules should trigger a large variation in the rate of reaction, with this issue being of particular relevance for high substrate concentrations (i.e. $\Phi$ should take low values for low $C^{*}$ and high values for high $C^{*}$ ). These postulates are consistent with the following assumption

$$
\Phi\left\{C^{*}\right\}=C^{* m}=\frac{1}{\phi\left\{C^{*}\right\}} \quad(m>0)
$$

where $m$ is a parameter yet to be determined.

Finally, each univariate function playing the role of the intrinsic contribution of $r^{*}$ and $r^{\prime *}$ in $f$ may in principle be expanded as a Taylor series, or equivalently, 
be described by an integer power series of the type

$$
\begin{gathered}
\psi\left\{r^{*}\right\}=\sum_{i=0}^{\infty} a_{i} r^{* i} \\
\Psi\left\{r^{\prime *}\right\}=\sum_{i=0}^{\infty} b_{i} r^{\prime * i}
\end{gathered}
$$

where the $a_{i}$ s and the $b_{i}$ s denote real coefficients yet to be determined.

Returning to equation (4), one is dealing with a typical problem of calculus of variations (or theory of functionals). The necessary condition that $r^{*}$ must satisfy in order to $I$ yield a stationary value is given by Euler's equation [4]:

$$
\left(\frac{\partial f}{\partial r^{*}}\right)_{C^{*}, r^{\prime *}}-\frac{\mathrm{d}}{\mathrm{d} C^{*}}\left\{\left(\frac{\partial f}{\partial r^{\prime *}}\right)_{C^{*}, r^{*}}\right\}=0
$$

Combination of equations (5)-(8) gives

$C^{*-m}\left(\sum_{i=1}^{\infty} i a_{i} r^{* i-1}\right)-m C^{* m-1}\left(\sum_{i=1}^{\infty} i b_{i} r^{\prime * i-1}\right)-C^{* m} r^{\prime \prime *}\left(\sum_{i=2}^{\infty} i(i-1) b_{i} r^{* i-2}\right)=0$

where $r^{\prime \prime *}$ is the second derivative of $r^{*}$ with respect to $C^{*}$. Recalling equations (1)-(3), equation (9) finally becomes

$$
\left(\sum_{i=1}^{\infty} \frac{i a_{i} C^{* i-m-1}}{\left(1+C^{*}\right)^{i-1}}\right)-\left(\sum_{i=1}^{\infty} \frac{m i b_{i} C^{* m-1}}{\left(1+C^{*}\right)^{2 i-2}}\right)+\left(\sum_{i=2}^{\infty} \frac{2 i(i-1) b_{i} C^{* m}}{\left(1+C^{*}\right)^{2 i-1}}\right)=0
$$

Due to the open form of equation (10), solutions are to advantage obtained by selecting, at each time, the $i$ th term of the second summation and the $i$ th term of the third summation in equation (10) followed by equating such two terms with those remaining that exhibit the same functional form for the denominator, i.e. the $(2 i-1)$ th and the $(2 i)$ th terms, respectively, of the first summation in equation (10). Due to the sufficient condition for every term that must be met by two infinite series in order to be equal to one another, two equations result each time the aforementioned process is applied; one such equation encompasses the $a$ and $b$ multiplying coefficients, whereas the other encompasses the $m$ exponent coefficient. It is interesting to note that solutions of equation (10) can be found only for discrete values of $m$; the (positive) eigenvalues are of the general form $(2 n-1) / 2(n=2,3,4, \cdots)$. For each such eigenvalue, solution of equation (10) yields a particular solution (i.e. an eigenvector or eigenfunction), which is given by $a_{2 n-1=}(n / 2) b_{n}$ and $a_{2 n}=-(n-1) b_{n}$. The general form of function $f$ must then be written as the sum of all eigenvectors for each positive eigenvalue $m$, thus yielding

$$
f\left\{C^{*}, r^{*} r^{\prime *}\right\}=\sum_{n=2}^{\infty} b_{n}\left(C^{*-(2 n-1) / 2}\left(\frac{n}{2} r^{* 2 n-1}-(n-1) r^{* 2 n}\right)+C^{*(2 n-1) / 2} r^{* n}\right)
$$

which, in combination with equations (2) and (3), becomes

$$
f\left\{C^{*}, r^{*}, r^{*}\right\}=\sum_{n=2}^{\infty} b_{n}\left(\frac{n C^{*(2 n-1) / 2}}{2\left(1+C^{*}\right)^{2 n-1}}-\frac{(n-1) C^{*(2 n+1) / 2}}{\left(1+C^{*}\right)^{2 n}}+\frac{C^{*(2 n-1) / 2}}{\left(1+C^{*}\right)^{2 n}}\right)
$$

where $b_{n}$ denotes an arbitrary constant. In the absence of any particular rationale 


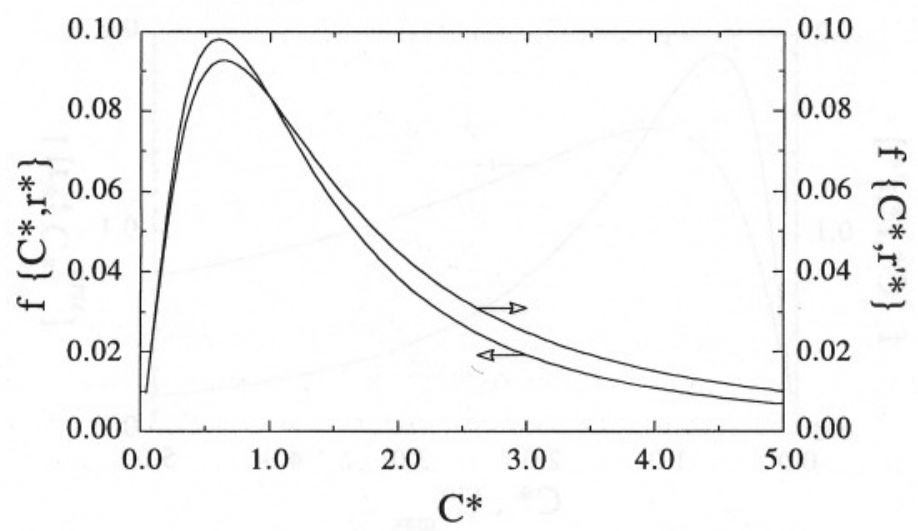

Figure 2. Variation of the partial dimensionless objective function relating to the activity of the enzyme, $f\left\{C^{*}, r^{*}\right\}$, and the partial dimensionless objective function relating to the controllability of the enzyme, $f\left\{C^{*}, r^{\prime *}\right\}$, versus the dimensionless substrate concentration, $C^{*}$.

for selecting different weights for different $n$ terms, the simplest (nontrivial) value of unity was considered hereafter for every constant $b_{n}$. The independent contributions arising from $r^{*}$ (i.e. $f\left\{C^{*}, r^{*}\right\}$, or the first two terms of the summation in equation (12)) and from $r^{\prime *}$ (i.e. $f\left\{C^{*}, r^{\prime *}\right\}$, or the last term of the summation in equation (12)) are plotted in Figure 2. The whole function $f\left\{C^{*}, r^{*}, r^{*}\right\}$ is depicted in Figure 3 .

Combination of equations (4) and (12), after appropriate change of variable to $C^{*}=\chi^{2}$, yields

$$
\max I\left[r^{*}\right]=\frac{2}{C_{\max }^{*}} \sum_{n=2}^{\infty} \int_{0}^{\sqrt{C_{\max }^{*}}}\left(\frac{n \chi^{2 n}}{2\left(1+\chi^{2}\right)^{2 n-1}}-\frac{(n-1) \chi^{2(n+1)}}{\left(1+\chi^{2}\right)^{2 n}}+\frac{\chi^{2 n}}{\left(1+\chi^{2}\right)^{2 n}}\right) \mathrm{d} \chi
$$

which, after some algebraic manipulation, becomes

$$
\max I\left[r^{*}\right]=\frac{2}{C_{\max }^{*}} \sum_{n=2}^{\infty} \int_{0}^{\sqrt{C_{\max }^{*}}}\left(\frac{(1+(n / 2)) \chi^{2 n}+(1-(n / 2)) \chi^{2(n+1)}}{\left(1+\chi^{2}\right)^{2 n}}\right) \mathrm{d} \chi
$$

and finally, after suitable expansion in partial fractions, gives

$$
\max I\left[r^{*}\right]=\frac{2}{C_{\max }^{*}} \sum_{n=2}^{\infty} \sum_{i=1}^{2 n}\left(\int_{0}^{\sqrt{C^{*} \max }} \frac{A_{i}+B_{i} \chi}{\left(1+\chi^{2}\right)^{i}} \mathrm{~d} \chi\right)
$$

where the $A_{i} \mathrm{~s}$ and the $B_{i} \mathrm{~s}$ are real constants. As a consequence of the even nature of the exponents of $\chi$ and the absence of zero order terms in equation (14), it can be easily shown that all $B_{i}$ s are zero. The terms $A_{i}$ may then be determined by simultaneous solution of the following set of linear algebraic equations

$$
\sum_{i=1}^{2 n}\left(\sum_{j=0}^{2 n-i}\left(\begin{array}{c}
2 n-i \\
j
\end{array}\right) \chi^{2 j}\right) A_{i}=\left(1+\frac{n}{2}\right) \chi^{2 n}+\left(1-\frac{n}{2}\right) \chi^{2(n+1)} \quad n=2,3, \cdots
$$




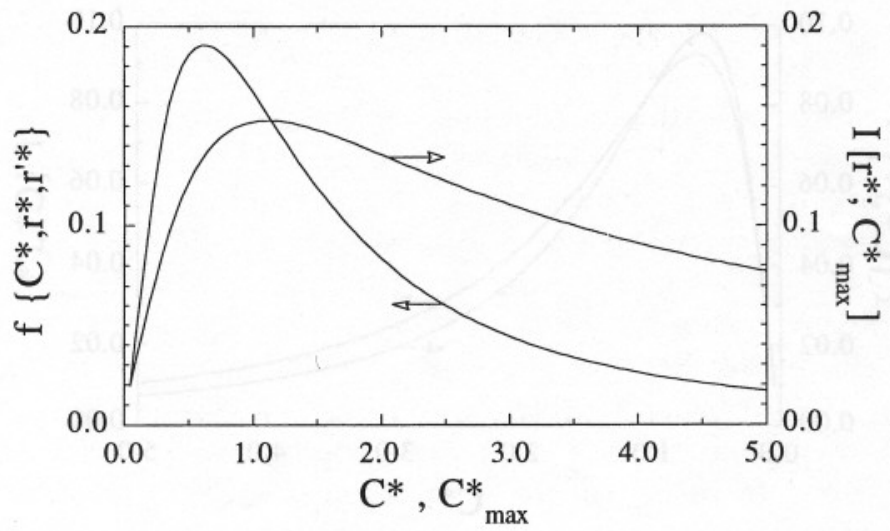

Figure 3. Variation of the dimensionless objective function, $f\left\{C^{*}, r^{*}, r^{\prime *}\right\}$, and the dimensionless integral thereof, $I\left[r^{*}\right]$, versus the dimensionless substrate concentration, $C^{*}$, or versus the upper limit of the dimensionless substrate concentration, $C_{\max }^{*}$.

where advantage should be taken from the following recurrence relation [5]

$$
\begin{aligned}
& \int_{0}^{\sqrt{C^{*} \max }} \frac{A_{i}}{\left(1+\chi^{2}\right)^{j}} \mathrm{~d} \chi \\
& \quad=\frac{A_{i} \sqrt{C_{\max }^{*}}}{2(j-1)\left(1+C_{\max }^{*}\right)^{j-1}}+\frac{2 j-3}{2(j-1)} \int_{0}^{\sqrt{C_{\max }^{*}}} \frac{A i}{\left(1+\chi^{2}\right)^{j-1}} \mathrm{~d} \chi \quad \begin{array}{l}
i=1,2, \ldots, 2 n \\
j=2,3, \ldots, i
\end{array}
\end{aligned}
$$

coupled with

$$
\int_{0}^{\sqrt{C_{\max }^{*}}} \frac{A_{i}}{1+\chi^{2}} \mathrm{~d} \chi=A_{i} \operatorname{arctg}\left\{\sqrt{C_{\max }^{*}}\right\} \quad i=1,2, \ldots, 2 n
$$

The optimum value of $I$, and the corresponding optimal value of $f$, as depicted in equation (15), are plotted versus $C_{\max }^{*}$, or $C^{*}$, in Figure 3.

\section{Discussion}

It should be emphasized that the objective function obtained is not the only function whose integral is maximized by the Michaelis-Menten rate expression; in fact, a multitude of objective functions, e.g. $f\left\{C^{*}, r^{*}, r^{*}\right\}=\left(1+C^{*}\right) r^{* 2}+r^{\prime *} C^{* 2}$, can be derived that also meet the aforementioned criterion. However, justification of the mathematical form of most of these alternative functions is much more difficult than of the one considered, which makes them of lesser discussion interest.

The strategy of solution followed in the problem developed above is inspired, to some extent, by the Frobenius method for the series solution of linear secondorder ordinary differential equations (where each actual value of parameter $m$ results from solution of the indicial equation that is obtained from equation (10) for each integer power of $r^{\prime *}$ ), on the one hand, and by the method of separation of variables for the solution of linear second-order partial differential equations (where particular solutions are obtained in series form which, although not sufficiently general to completely fulfil the goals of a pure mathematician, allow 
one to be directed by the type of boundary conditions to be imposed), on the other hand.

It is noteworthy that the contributions of $r^{*}$ and $r^{*}$ to the objective function $f$ (i.e. $f\left\{C^{*}, r^{*}\right\}$ and $f\left\{C^{*}, r^{*}\right\}$, respectively) are quite similar (see Figure 2), and this observation suggests that the maximum of $I$ for each selected value of $C_{\max }^{*}$ is actually obtained from balanced contributions of $r^{*}$ and $r^{\prime *}$. Therefore, one would expect (as observed in Figure 3) that the whole objective function $f\left\{C^{*}, r^{*}, r^{*}\right\}$ would exhibit the same shape as its individual components $f\left\{C^{*}, r^{*}\right\}$ and $f\left\{C^{*}, r^{\prime *}\right\}$. It is remarkable that, although the maximum value of $f$ is achieved at $C^{*}=0.6$, the highest value of $I$ (i.e. the average of $f$ over the whole concentration range) is obtained in the vicinity of $C_{\max }^{*}=1$ (see Figure 3), or, recalling equation (1), when $C=K_{\mathrm{m}}$. Inspection of classical literature (e.g. [1]) indicates that there is a dominating tendency for cells to operate at intracellular concentrations that are usually not much above the Michaelis-Menten constant, say $10^{-2}$ to $10 \mathrm{~mol} \mathrm{~m}^{-3}[3]$; hence mathematical confirmation that nature has followed optimal conditions in terms of an average behaviour is apparent.

\section{Notation}

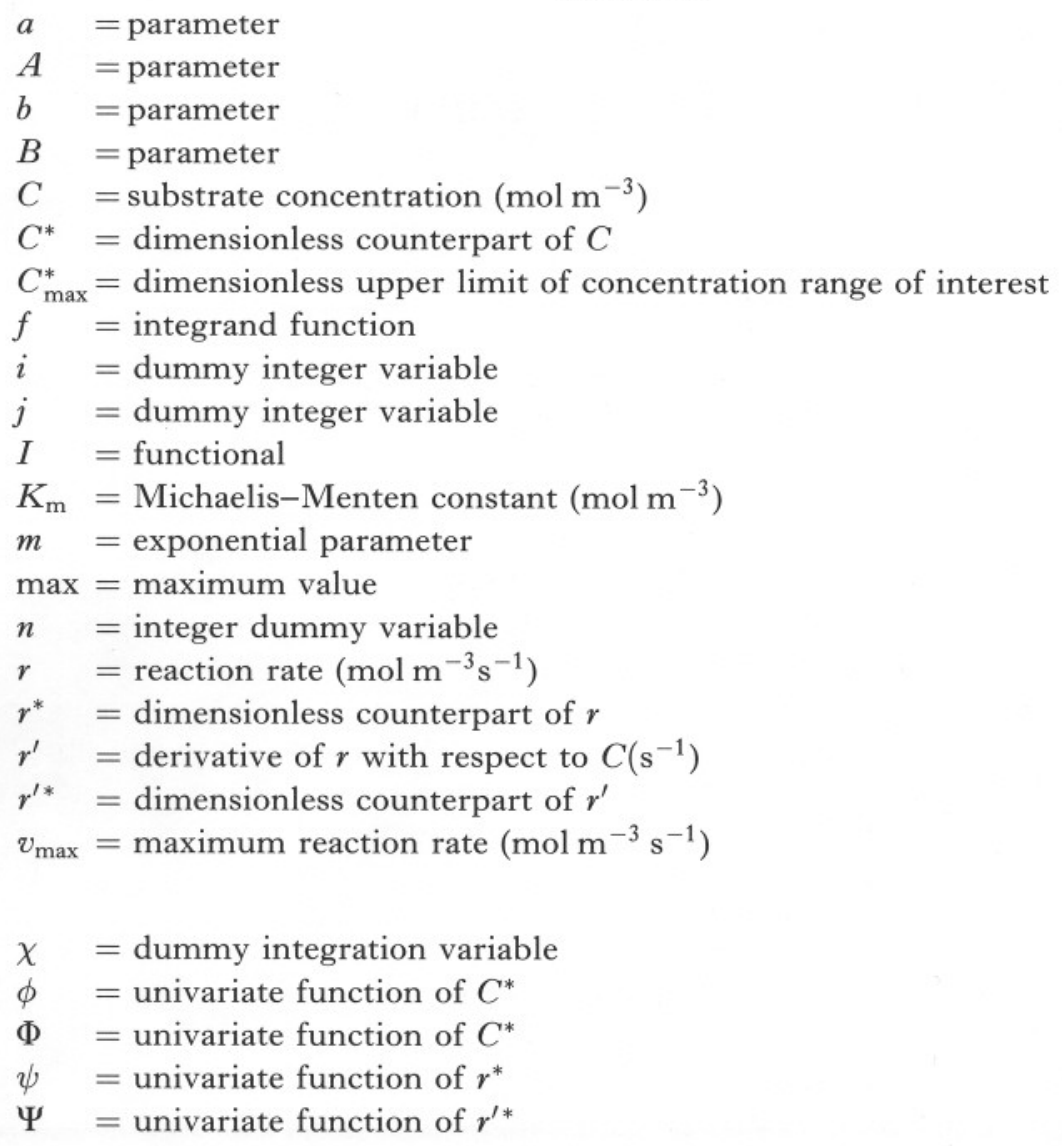




\section{References}

[1] Bailey, J. E., and Ollis, D. F., 1986, Biochemical Engineering Fundamentals (New York: McGraw-Hill).

[2] Whitaker, J.R., 1972, Principles of Enzymology for the Food Science (New York: Marcel Dekker).

[3] Segel, I.H, 1975, Enzyme Kinetics: Behaviour and Analysis of Rapid Equilibrium and Steady-state Enzyme Systems (New York: Wiley).

[4] Stephenson, G., 1973, Mathematical Methods for Science Students (London: Longman).

[5] Gradsteyn, I.S., and Ryzhiк, I.M., 1980, Table of Integrals, Series and Products (San Diego: Academic Press). 